\title{
Assessment of Right Ventricular Oxidative Metabolism by Positron Emission Tomography with C-11 Acetate in Aortic Valve Disease
}

\author{
Rodney J. Hicks, MB, BS, Victor Kalff, MB, BS, Vicky Savas, MD, \\ Mark R. Starling, MD, and Markus Schwaiger, MD
}

\begin{abstract}
Evaluation of right ventricular (RV) oxidative metabolism is limited by the inability to easily determine oxygen extraction by the RV myocardium and the complex morphology of this ventricle. Because left ventricular C-11 clearance rate constants closely correlate with myocardial oxygen consumption, it was postulated that C-11 clearance rate constants for the RV free wall should also reflect its oxygen consumption. Therefore, RV C-11 clearance rate constants were compared with RV loading in 21 patients with aortic valve disease to assess the possible use of this technique for noninvasive evaluation of RV oxidative metabolism. RV free wall C-11 clearance rate constants correlated with the product of systolic pulmonary artery pressure and heart rate for all patients $(r=0.65, p=$ 0.002 ), but the relation was stronger if 2 patients with overt RV dysfunction were excluded $(r=0.83$, $p=0.001$ ). On the basis of mean pulmonary artery pressures, patients were stratified into subgroups with normal (group I, $\mathbf{n}=\mathbf{8}$ ) and elevated (group II, $n=13$ ) pulmonary pressures and were compared with 10 normal control subjects. RV C-11 clearance rate constants were significantly higher in group II than in group I and in normal control subjects $(p<0.05)$. These data suggest that RV C-11 acetate clearance rate constants can provide noninvasive evaluation of RV oxidative metabolism. This technique may allow serial assessment of RV performance in various cardiac and pulmonary diseases, and particularly of changes associated with therapeutic interventions.
\end{abstract}

(Am J Cardiol 1991;67:753-757)

From the Divisions of Nuclear Medicine and Cardiology, Department of Internal Medicine, at the University of Michigan Medical Center, Ann Arbor, Michigan. This study was supported in part by Grants RO1 HL41047-1 and HL36450 from the National Institutes of Health, Bethesda, Maryland, and performed under the tenurc of an cstablished investigatorship of the American Heart Association (MS), Dallas, Texas. Manuscript received October 10, 1990; revised manuscript received November 30, 1990, and accepted December 12.

Address for reprints: Markus Schwaiger, MD, Director of Nuclear Cardiology, Department of Nuclear Medicine, University of Michigan Hospitals, B1G412 Ann Arbor, Michigan 48109-0028.
I mpairment of right ventricular (RV) performance can cause significant morbidity in cardiac and pulmonary diseases. Right-sided cardiac catheterization, echocardiography and radionuclide ventriculography provide evaluation of RV loading and function. ${ }^{1,2}$ However, none of these techniques provides direct evaluation of RV oxidative metabolism either globally or regionally. Indeed, oxidative metabolism within the right ventricle has been only poorly characterized because of the inability to measure oxygen extraction by RV myocardium and the complex morphology of the right ventricle, which limits assessment of RV wall stress. ${ }^{3-8}$

The RV free wall makes an important contribution to RV performance ${ }^{9-12}$ and may be responsible for maintaining cardiac output in advanced LV dysfunction. Because metabolic demands placed on the RV free wall are influenced significantly by the performance of the ventricular septum, ${ }^{6}$ the ability to directly characterize oxidative metabolism regionally within the right ventricle would have significant advantages.

C-11 acetate has recently been introduced and validated as a tracer of myocardial oxidative metabolism for use in combination with positron emission tomography (PET).${ }^{13-16}$ We postulated that C-11 acetate clearance rate constants in the RV free wall would reflect its oxygen consumption and correlate with parameters of RV loading in the compensated right ventricle. Consequently, we evaluated the relation between RV C-11 acetate clearance rate constants and RV hemodynamic data in 21 patients with aortic valve disease. The aim of this study was to determine the possible use of this approach for the noninvasive evaluation of RV oxidative metabolism.

\section{METHODS}

Patients: The study protocol was approved by the Human Subject Protection Committee of the University of Michigan Medical Center. All normal subjects and patients were studied only after granting informed, written consent.

All 21 patients in the group had aortic valve disease documented by echocardiography and were being electively evaluated for possible aortic valve replacement. This population was chosen because such patients generally have only modest elevation of pulmonary artery pressures and well-preserved RV contractile function. 


\begin{tabular}{|lll|}
\hline \multicolumn{3}{|l|}{ TABLE I Patient Characteristics } \\
\hline & $\begin{array}{l}\text { Group I } \\
(\mathrm{n}=8)\end{array}$ & $\begin{array}{l}\text { Group II } \\
(\mathrm{n}=13)\end{array}$ \\
\hline $\begin{array}{c}\text { Mean Age } \pm \mathrm{SD} \\
\text { (years) }\end{array}$ & $61 \pm 16$ & $65 \pm 7$ \\
Age range (years) & $38-35$ & $50-75$ \\
Men:women & $6: 2$ & $7: 6$ \\
Primary diagnosis & $3 \mathrm{AR}, 5 \mathrm{AS}$ & $6 \mathrm{AR}, 4 \mathrm{AS}, 3$ mixed \\
\hline AR = aortic regurgitation; AS $=$ aortic stenosis; mixed $=$ mixed aortic and mitral \\
valve disease; SD = standard deviation.
\end{tabular}

Eighteen patients had isolated aortic valve disease and 3 patients had mixed mitral and aortic valve disease. All were clinically stable, although 2 had chronic RV failure. None had significant coronary artery disease based on coronary arteriography performed within 1 month of entry into the study. These patients were compared with a group of 9 healthy male volunteers, 22 to 29 years of age (mean \pm standard deviation $26 \pm 3$ ). All volunteers were nonsmokers and were considered to have a low likelihood of coronary artery disease or other cardiac disease based on age, clinical history, physical examination and the presence of a normal electrocardiogram at rest.

Right ventricular hemodynamic data: Right-sided cardiac catheterization was performed for clinical indications in all patients but not in the normal volunteers. RV hemodynamic data were obtained within 1 month of PET and without change in medication in the study interval. Mean, systolic and diastolic pulmonary artery pressures were measured using standard techniques and with the patient lying quietly in the supine position. A RV rate-pressure product was derived by multiplying the systolic pulmonary artery pressure at the time of

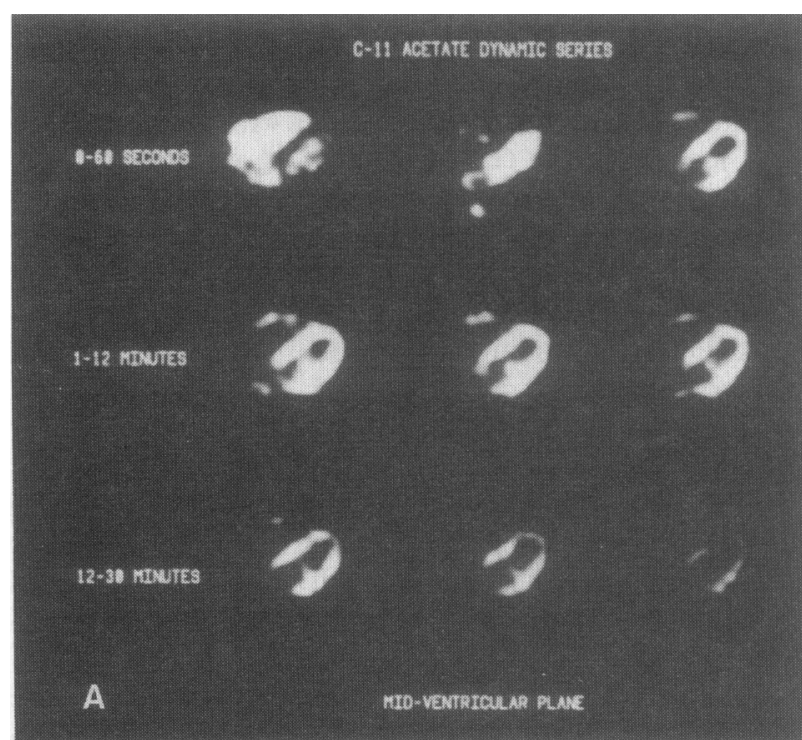

right-sided cardiac catheterization by the heart rate recorded at the time of the PET study.

Patients were stratified into subgroups with normal (group I, $\mathrm{n}=8$ ) and elevated (group II, $\mathrm{n}=13$ ) pulmonary artery pressures based on the recorded mean pulmonary artery pressure. A mean pulmonary artery pressure $>20 \mathrm{~mm} \mathrm{Hg}$ was considered pathologically elevated. ${ }^{17}$ The age, sex and clinical characteristics of these subgroups of patients are listed in Table I.

C-11 acetate positron emission tomography: C-11 acetate PET studies were performed using a whole body PET scanner (Siemens 931, CTI, Knoxville, Tennessee), allowing simultaneous imaging of 15 transaxial slices, $6.75 \mathrm{~mm}$ in thickness. Transmission images were obtained for 15 minutes and were used for attenuation correction. Dynamic PET imaging was then performed for 31 minutes after intravenous administration of 740 $\mathrm{MBq}(20 \mathrm{mCi})$ of $\mathrm{C}-11$ acetate. Ten frames of $90 \mathrm{sec}-$ onds' duration were obtained, followed by 5 frames of 120 seconds' duration and 2 frames of 180 seconds' duration. C-11 acetate was synthesized using previously described methods. ${ }^{13}$

A single midventricular transverse, transaxial plane in which the right ventricle could be easily identified was chosen for analysis. The decay-corrected, dynamic series of 17 C-11 acetate images for this plane was displayed and regions of interest were assigned on the frame with best myocardial definition (Figure 1A). Regions of interest were assigned for the RV free wall and septum and were then extrapolated to all frames. Monoexponential fitting of the resultant regional timeactivity curves was performed after decay correction (Figure 1B). Clearance half-times $\left(\mathrm{t}^{1 / 2} / 2\right)$ were obtained and $\mathrm{C}-11$ acetate rate constants $(\mathrm{k} / \mathrm{min})$ were calculated by dividing the natural logarithm of 2 by the $\mathrm{C}-11$ acetate clearance $t^{1 / 2}$ in minutes, i.e. $k=\ln 2 / t^{1 / 2} .^{18}$

Statistical analysis: Values for groups are presented as mean \pm standard deviation. Mean levels of continu-

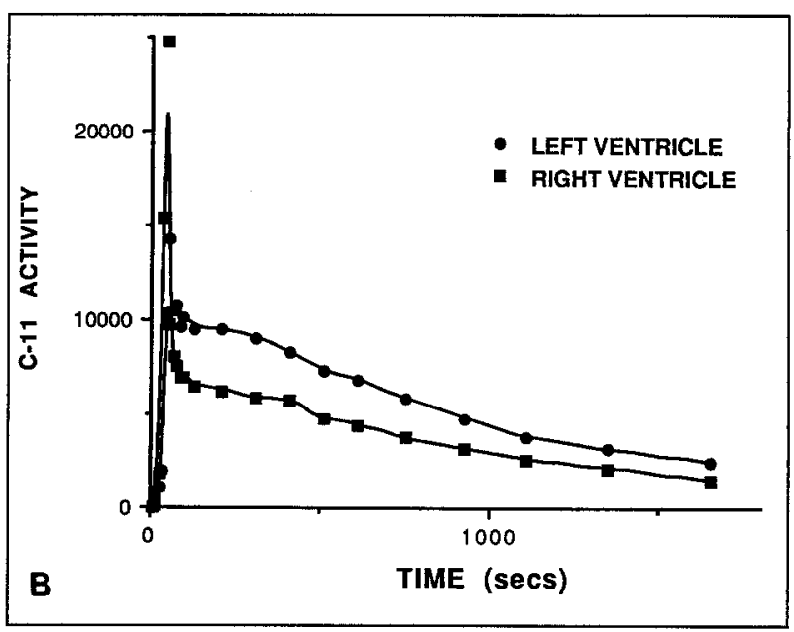

FIGURE 1. A, dynamic series of decay-corrected, C-11 acetate images for a single midventricular, transaxial plane. In the first frame (top left), C-11 activity is mainly in blood. Later, there is rapid and marked blood pool clearance and avid myocardial extraction. By the final frame (bottom right) there has been substantial clearance of myocardial C-11 activity, reflecting metabolism of C-11 acetate via the tricarboxylic acid cycle. B, decay-corrected time-activity curves for right ventricular and left ventricular myocardial regions of interest. Data presented in this figure were derived from analysis of a study done in a patient with elevated pulmonary artery pressures secondary to aortic regurgitation. 
ous factors were compared between groups using an unpaired $t$ test. RV and septal C-11 acetate clearance rate constants were compared in each patient using a Student's $t$ test for paired data. Multiple regression analysis was performed to evaluate the relation between $\mathrm{RV}$ hemodynamic data and the RV free wall and septal C-11 acetate clearance rate constants. Pearson's correlations were used to test the association between 2 continuous factors. Probability levels of $<0.05$ were considered statistically significant.

\section{RESULTS}

Comparison of C-11 acetate clearance with right ventricular hemodynamic data: RV C-11 clearance rate constants were significantly correlated with the RV rate-pressure product for all patients (Figure 2A). The 2 patients in this study with overt evidence of RV fail-
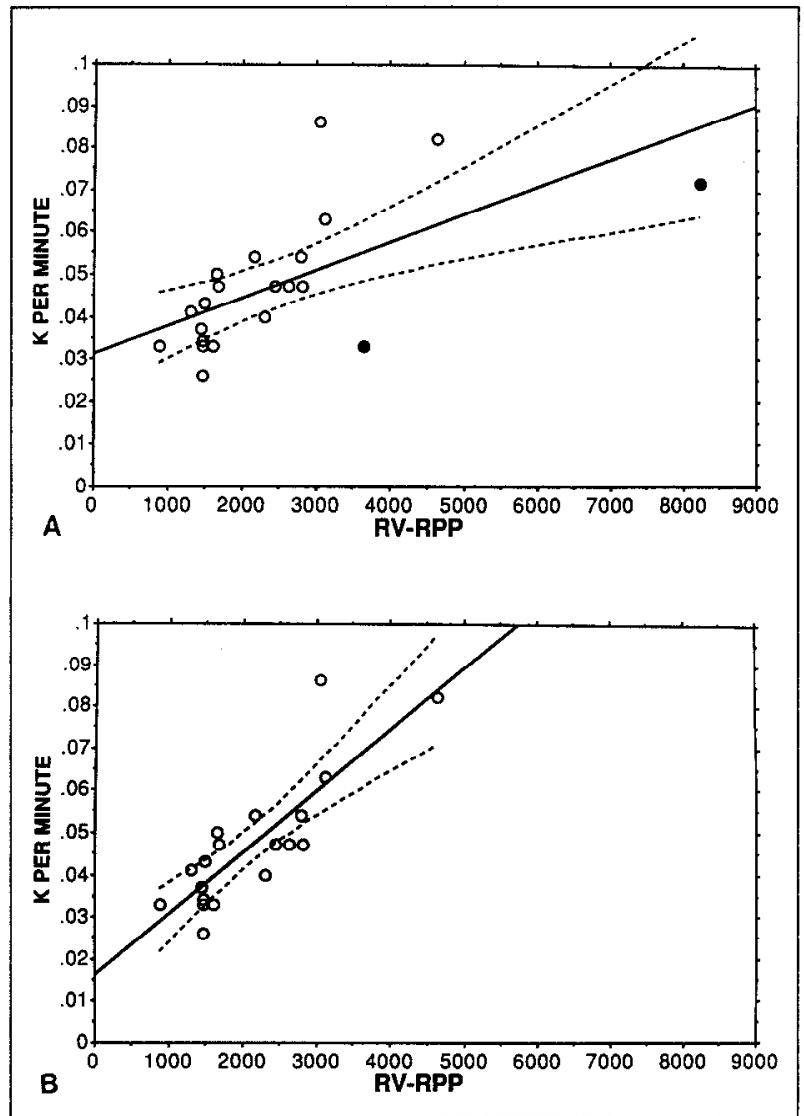

FIGURE 2. A, plot of right ventricular (RV) C-11 clearance rate constants versus the $R V$ rate-pressure product (RPP) calculated by multiplying the systolic pulmonary artery pressure at the time of right-sided cardiac catheterization and heart rate during the positron emission study. A significant correlation was noted $(r=0.65, p=0.002)$. Two patlents had overt RV dysfunction (closed circles) included in this regression. The $95 \%$ confidence bands of the true mean of acetate rate constants $(\mathbf{k})$ per minute are displayed. $B$, exclusion of the 2 patients with RV dysfunction who likely had impaired contractility made the correlation between these parameters stronger $(r=0.83, p=0.001)$. Again, the $95 \%$ confidence bands of the true mean of $k$ per minute are displayed. These data suggest that C-11 clearance rate constants are closely related to RV loading in the compensated right ventricle but less well in the failing right ventricle. ure lay well below the line of identity for this relation. If these patients with RV decompensation and probable impairment of RV contractility were excluded from analysis, a stronger correlation was observed between $\mathrm{C}-11$ clearance in the RV free wall and the RV ratepressure product $(r=0.83, p=0.001$ ) (Figure $2 B$ ).

Mean pulmonary artery pressures were significantly higher in the patient subgroup defined as having elevated pulmonary artery pressures (group II) than in the subgroup defined as having normal pulmonary artery pressures (group I) $(28 \pm 12$ vs $13 \pm 2 \mathrm{~mm} \mathrm{Hg}, \mathrm{p}$ $<0.005$, respectively). RV C-11 acetate clearance rate constants were significantly higher in group II than in group I patients $(\mathrm{p}<0.05)$ and in normal subjects $(\mathrm{p}$ $<0.005$ ) (Figure 3). C-11 clearance was faster in patients in group I than in normal subjects without evidence of cardiac disease, although the difference was not statistically significant.

Comparison of right ventricular and septal C-11 clearance kinetics: In patients and in normal volunteers, C-11 acetate clearance rate constants were significantly higher in the septum than in the RV free wall $(0.064 \pm 0.013$ vs $0.043 \pm 0.016, p=0.0001)$. However, C-11 acetate clearance rate constants in the RV free wall correlated with those in the septum $(r=0.75$, $p=0.0001)$. Despite this relation, linear regression analysis of $\mathrm{C}-11$ acetate clearance rate constants in the septum versus $R V$ hemodynamic parameters revealed no significant correlation except with heart rate $(r=$ $0.53, \mathrm{p}<0.05)$. This was in contrast to the RV free wall C-11 acetate clearance rate constants which correlated significantly with mean pulmonary artery pressure $(\mathrm{r}=$ $0.62, \mathrm{p}<0.005$ ), systolic pulmonary artery pressure ( $\mathrm{r}$ $=0.56, \mathrm{p}<0.01), \mathrm{RV}$ rate-pressure product $(\mathrm{r}=0.65, \mathrm{p}$ $<0.005)$ and heart rate $(\mathrm{r}=0.47, \mathrm{p}<0.05)$. Multiple regression analysis demonstrated a significant correlation between $\mathrm{RV}$ free wall $\mathrm{C}-11$ acetate clearance rate

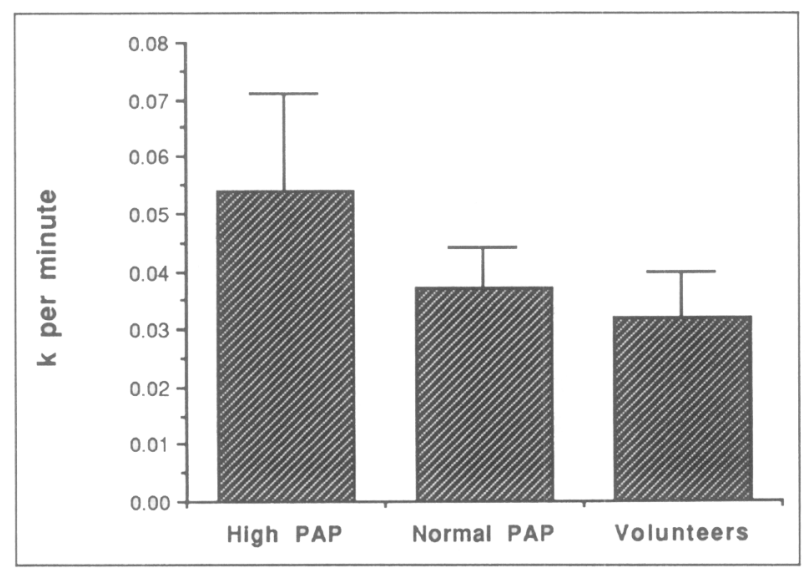

FIGURE 3. C-11 acetate clearance rate constants were, on average, significantly higher in 13 patients with elevated pulmonary artery pressure (PAP) than in 8 patients with normal pulmonary artery pressures and in 10 normal subjects (Volunteers), C-11 clearance was also slightly faster in patients with normal pulmonary artery pressures than in normal volunteers, possibly refiecting the fact that mean pulmonary artery pressures in this group of patients were in the upper end of the normal range. 
constants and 5 potential predictors of RV oxygen demand including mean, systolic and diastolic pulmonary artery pressures, and RV rate-pressure product and heart rate (cumulative $r=0.8, p=0.006$ ). Similar comparison between $\mathrm{C}-11$ acetate clearance rate constants in the septum and the same 5 parameters revealed no significant correlation (cumulative: $r=0.6$, $\mathrm{p}=0.17$ ).

Comparison of each study subgroup showed that the ratio of $\mathrm{RV}$ free wall to septal C-11 acetate clearance rate constants was significantly higher in group II than in group $\mathrm{I}(78 \pm 15 \%$ vs $57 \pm 8 \%, \mathrm{p}<0.005)$ and in normal volunteers $(78 \pm 15 \%$ vs $58 \pm 10 \%$, p <0.005). Also, although there was a significant difference in RV free wall C-11 acetate clearance rate constants in group II compared with group I $(0.054 \pm 0.017$ vs $0.037 \pm$ $0.007, p=0.003$ ), there was no significant difference in C-11 clearance in the septum of the 2 subgroups $(0.069$ \pm 0.014 vs $0.065 \pm 0.011$ ).

\section{DISCUSsION}

C-11 acetate has been validated as a tracer of myocardial oxidative metabolism in both animals ${ }^{16,19,20}$ and humans. ${ }^{21}$ We have previously shown the unique ability of $\mathrm{C}-11$ acetate kinetics to provide noninvasive evaluation of regional LV oxidative metabolism. ${ }^{22}$ Based on these findings, we hypothesized that analysis of RV free wall C-11 acetate clearance kinetics should provide noninvasive evaluation of RV oxidative metabolism.

Our primary hypothesis could not be directly validated because of the inability to measure RV oxygen consumption directly. However, the positive correlation between RV free wall C-11 acetate clearance rate constants and RV hemodynamic data demonstrated in this study supports our hypothesis since increased loading conditions would be predicted to increase RV oxygen demand. Furthermore, lower C-11 acetate clearance rate constants were observed in the RV free wall than in the septum in both normal subjects and patients. This is consistent with the lower stroke work of the right ventricle compared with the left. There was a significant correlation between $\mathrm{C}-11$ clearance rate constants in the RV free wall and septum ( $r=0.75, p=0.0001$ ) supporting the functional interdependence of these regions. ${ }^{6}$ However, C-11 clearance rate constants correlated with pulmonary hemodynamics, whereas those in the septum did not. These data further suggest that regional RV clearance of $\mathrm{C}-11$ acetate reflects local oxygen demand.

Pathophysiologic considerations: Myocardial oxygen consumption is determined by energy demand, which integrates the effects of myocyte loading, and the rate of isometric force production. Consequently, changes in cardiac performance that occur in pressure and volume overload of the right ventricle may alter the relation between loading conditions and oxygen demand. ${ }^{23}$ In compensated RV hypertrophy resulting from pressure overload, performance of the right ventricle as assessed from pressure measurements may appear to be elevated but is essentially normal when corrected for volume ${ }^{24}$ or RV mass. ${ }^{25}$ However, depressed RV contractility would be expected to decrease oxygen consumption relative to a right ventricle with normal con- tractile function under the same loading conditions. Assessment of RV loading alone, particularly in the failing ventricle, may give inadequate evaluation of myocardial oxidative metabolism. Under these conditions, C-11 acetate clearance kinetics that provide direct assessment of myocardial oxygen consumption, integrating both loading determinants of myocardial oxygen demand and the effects of altered contractility, may provide unique characterization of RV performance.

The scatter about the line of identity relating RV C11 acetate clearance rate constants and RV rate-pressure products in our study group (Figure 2A) may well reflect the limitations of RV hemodynamics as predictors of RV free wall oxygen consumption rather than limitations of $\mathrm{C}-11$ acetate kinetics in assessing regional oxidative metabolism. This was evidenced by the improved correlation between $\mathrm{C}-11$ acetate clearance rate constants and the RV rate-pressure product when the 2 patients with RV failure were excluded from analysis (Figure 2B).

Study limitations: The major limitation of this study is the fact that RV hemodynamic data, with which C11 acetate clearance rate constants were compared, were obtained at a different time than the PET study. Although these patients were clinically stable, had no change in medication in the study interval, and underwent both studies under similar conditions, the possibility that there might have been fluctuation in pulmonary artery pressures cannot be excluded. Standard right-sided cardiac catheterization was performed in all patients as part of routine clinical management. Consequently, more sophisticated evaluation of RV loading including pressure-volume loops ${ }^{26}$ was not performed. These preliminary feasibility results in humans suggest that further assessment of the utility of PET with C-11 acetate is warranted in subgroups of patients with more advanced $R V$ disease.

The RV free wall thickness is generally much less than the intrinsic resolution of the PET device used (8 to $10 \mathrm{~mm}$ ). Nevertheless, correction for partial volume effects was not needed because C-11 acetate clearance rate constants are a rate of change in activity rather than an absolute measure of tissue activity and are thus insensitive to partial volume effects. A more important theoretical problem with these studies is the spillover of blood pool activity into the myocardial region of interest. Although C-11 acetate activity clears rapidly and substantially from the blood, ${ }^{14}$ the thin free wall of the right ventricle and the large adjacent blood pool make the effects of spillover relatively greater for the right ventricle than for the left ventricle. ${ }^{27}$ Without accurate measurement of $\mathrm{RV}$ wall thickness and chamber diameters, correction of cross-contamination of recorded counts between blood and myocardium was not possible. However, the effects of spillover on C-11 clearance rate constants are likely to have been minor since fitting of the myocardial time activity was performed after the blood pool activity had largely cleared and was relatively stable.

Clinical implications: The unique ability of PET with $\mathrm{C}-11$ acetate to provide noninvasive assessment of regional myocardial oxidative metabolism may provide 
an attractive option for following patients with RV volume or pressure overload. Assessment of the success of medications in decreasing RV oxygen demand could be assessed, and the combination of noninvasive evaluation of RV size and performance obtained by echocardiography and PET with C-11 acetate could provide evaluation of the progression and status of RV dysfunction in various cardiac and pulmonary diseases. The findings of this study warrant application of this approach to diseases, such as primary pulmonary hypertension, scleroderma, and mitral valve disease, which are more frequently associated with RV dysfunction than is aortic valve disease.

Acknowledgment: We thank Jill Rothley, CNMT, Leslie Shaw, CNMT, Annette Bethley, CNMT, Vincent McCormick, CNMT, for their technical assistance and the cyclotron and radiochemistry staff for preparing C-11 acetate.

\section{REFERENCES}

1. Haines DE, Beller GA, Watson DD, Nygaard TW, Craddock GB, Cooper AA, Gibson RS. A prospective clinical, scintigraphic, angiographic and functional evaluation of patients after inferior myocardial infarction with and without right ventricular dysfunction. J Am Coll Cardiol 1985;6:995-1003.

2. Manno BV, Iskandrian AS, Hakki AH. Right ventricular function: methodologic and clinical considerations in noninvasive scintigraphic assessment. $J \mathrm{Am}$ Coll Cardiol 1984;3:1072-1081.

3. Rushner RF, Crystal DK, Wagner C. The functional anatomy of ventricular contraction. Circ Res 1953;1:162-170.

4. Armour JA, Pace JB, Randall WC. Interrelationship of architecture and function of the right ventricle. Am J Physiol 1970;218:174-179.

5. Raines RA, LeWinter MM, Covell JM. Regional shortening patterns in canine right ventricle. Am J Physiol 1976;231:1395-1400

6. Santamore WP, Lynch PR, Heckman JL, Bove AA, Meier GD. Left ventricular effects on right ventricular developed pressure. J Appl Physiol 1976;41: 925-930.

7. Meier GD, Bove AA, Santamore WP, Lynch P. Contractile function in the canine right ventricle. Am J Physiol 1980:239:H794-H804.

8. Pouleur H, Lefevre J, Van Mechelen H, Charlier AA. Free wall shortening and relaxation during ejection in the canine right ventricle. Am J Physiol 1980; 239:H601-H613.

9. Agarwal JB, Yamazaki H, Bodenheimer MM, Banka VS, Helfant RH. Effects of isolated interventricular septal ischemia on global and segmental function of the canine right and left ventricle. Am Heart $J$ 1981:102:654-658.

10. Damiano RJJ, Asano T, Smith PK, Ferguson TB, Cox JL. Hemodynami consequences of right ventricular isolation: the contribution of the right ventricular free wall to cardiac performince. Ann Thorac Surg 1988;46:324-330.

11. Goldstein JA, Barzilai B, Rosamond TL, Eisenberg PR, Jaffe AS. Determinants of hemodynamic compromise with severe right ventricular infarction. Circulation 1990;82:359-368.

12. Tani $M$. Roles of the right ventricular free wall and ventricular septum in right ventricular performance and influence of the parietal pericardium during right ventricular failure in dogs. Am J Cardiol 1983;52:196-202.

13. Pike VW, Eakins MN, Allan RM, Selwyn AP. Preparation of [1-11C] acetate-an agent for the study of myocardial metabolism by positron emission tomography. Int J Appl Radiat Isot 1982;33:505-512.

14. Brown $M$, Marshall $D R$, Sobel BE, Bergmann SR. Delineation of myocardia oxygen utilization with carbon-11-labeled acetate. Circulation 1987;76:687-696. 15. Brown MA, Myears DW, Bergmann SR. Noninvasive assessment of canine Inyocardial uxidative metabolism with carbon-11 acetate and positron emission tomography. J Am Coll Cardiol 1988;12:1054-1063.

16. Buxton DB, Schwaiger M, Nguyen A, Phelps ME, Schelbert HR. Radiolabeled acetate as a tracer of myocardial tricarboxylic acid cycle flux. Circ Res 1988;63:628-634.

17. Grossman W, Alpert JS, Braunwald E. Pulmonary hypertension. In: Braunwald E, ed. Heart Disease. A Textbook of Cardiovascular Medicine. Philadelphia WB Saunders, 1984:823-848.

18. Armbrecht JJ, Buxton DB, Brunken RC, Phelps ME, Schelbert HR. Regional myocardial oxygen consumption determined noninvasively in humans with [1$11 \mathrm{C}]$ acetate and dynamic positron tomography. Circulation 1989;80:863-872 19. Brown MA, Myears DW, Bergmann SR. Validity of estimates of myocardial oxidative metabolism with carbon-11 acetate and positron emission tomography despite altered patterns of substrate utilization. J Nucl Med 1989;30:187-193 20. Buxton DB, Nienaber CA, Luxen A, Ratib O, Hansen $H$, Phelps ME Schelbert HR. Noninvasive quantitation of regional myocardial oxygen consumption in vivo with [1-1IC] acetate and dynamic positron emission tomography. Circulation 1989:79:134-142.

21. Henes CG, Bergmann SR, Walsh MN, Sobel BE, Geltman EM. Assessmen of myocardial oxidative metabolic reserve with positron emission tomography and carbon-11 acetate. $J$ Nucl Med 1989;30:1489-1499.

22. Kotzerke J, Hicks RJ, Wolfe E, Herman WH, Molina E, Kuhl DE Schwaiger M. Three-dimensional assessment of myocardial oxidative metabolism: a new approach for regional determination of PET-derived $\mathrm{C}-11$ acetate kinetics. J Nucl Med 1990;31:1876-1893.

23. Alpert NR, Mulieri LA. Heat, mechanics, and myosin ATPase in normal and hypertrophied heart muscle. Fed Proc 1982;41:192-198.

24. Wroblewski E, James F, Spann JF, Bove AA. Right ventricular performance in mitral stenosis. Am J Cardiol 1981;47:51-55.

25. Wyse RK, Jones M, Welham KC, de Leval MR. Cardiac performance and myocardial blood flow in pigs with compensated right ventricular hypertrophy. Cardiovasc Res 1984;18:733-745.

26. Friedman BJ, Lozner EC. Curfman GD, Herzberg D, Rolett EL. Characterization of the human right ventricular pressure-volume relation: effect of dobutamine and right coronary artery stenosis. I Am Coll Cardiol 1984;4:999-1005 27. Henze E, Huang SC, Ratib O, Hoffman E, Phelps ME, Schelbert HR. Measurements of regional tissue and blood-pool radiotracer concentrations from serial tomographic images of the heart. $J$ Nucl Med 1983;24:987-996. 